\title{
II Sinodo dei Vescovi nella Chiesa postconciliare (sintesi)
}

\section{The Synod of Bishops in the Post-Conciliar Church}

\begin{abstract}
The history of the Church for the past twenty centuries was moulded not only by the authorities of Church offices, such as the office of Bishop of Rome or the office of the diocesan bishop but also by the personal authority of the saints from various nations.

The Second Vatican Council (1962-1965) was an important period for the reflection of the Church on itself and its mission in the service of the Gospel. Due to its discussion this Council considerably contributed to establishing the institution of the Synod of Bishops by Paul VI on 15 September 1965. This took place during the last session of the conciliar debates.

After the completion of the Second Vatican Council, the Synod of Bishops has remained an institution which originates from the Council and shapes the image of the Church confirmed by the contemporary post-conciliar period and meetings. That is why one of the most crucial contributions of the Second Vatican Council to the organizational structure and the method of management of the Church is undoubtedly the Synod of Bishops, which is confirmed by the passing post-conciliar period and meetings of this institution whose number equals 24 .

Simultaneously, the Synod of Bishops played and has still been playing a crucial role also in the pontificates of the post-conciliar Popes, particularly during the years of the long-lasting pontificate of John Paul II (1978-2005). Furtthermore, the current pontificate indicates that Pope Benedict XVI attaches a high importance to this institution, summoning the Synod of Bishops regularly.
\end{abstract}


The afore-mentioned Popes of the post-conciliar Church modelled the theological and legal image of the Synod of Bishops as well. First of all, in this current the following should be listed: the before-mentioned m.p. „Apostolica sollicitudo" by Paul VI establishing the institution which has been remaining the theological and legal backbone of the Synod of Bishops till today and later, giving it the current legal depiction with division into the General Assembly of the Synod of Bishops (ordinary and extraordinary ones) and the Special Assembly of the Synod of Bishops promulgated by the Pope John Paul II in 1983 under the Code of Canon Law.

Furthermore, statutory regulations of this central institution of the Church which are the Regulations of the Synod of Bishops from the years: 1966, 1969, 1971 and 2006 should be mentioned. The Regulation of the Synod of Bishops has shaped the works of its individual meetings in a practical way. In this current we should also refer to multi-layered statements of the Bishops of Rome at numerous circumstances about the Synod of Bishops and their constant practice of issuing post-synodal apostolic exhortations.

\section{Keywords}

Bishop of Rome, Church, John Paul II, Regulation of the Synod of Bishops, Second Vatican Council, Synod of Bishops.

La storia della Chiesa, tramite gli ultimi venti secoli, è stata creata non soltanto dalle autorità ecclesiastiche come quella del Vescovo di Roma ovvero del vescovo diocesano, ma anche dalle autorità personali dei santi delle diverse nazioni o degli stati della Chiesa, ed anche dalle sue istituzioni. Tra queste ultime si deve nominare il Concilio ecumenico, la cui riunione ogni volta diventava un evento significativo dal punto di vista ecclesiastico, ma anche sociale, spesse volte iniziava una nuova tappa nella storia della Chiesa, come p.e. una profonda riforma della Chiesa e delle sue istituzioni dopo il Concilio di Trento.

Il Concilio Vaticano II (1962-1965) è stato un periodo assai significativo dedicato a una riflessione della Chiesa su se stessa e sulla sua missione al servizio del Vangelo. Proprio questo Concilio ha contribuito in modo significativo, tramite le discussioni, alla costituzione dell'istituzione del Sinodo dei Vescovi dal Papa Paolo VI addì 15 settembre 1965 cioè proprio durante i dibattiti del Concilio ${ }^{1}$.

\footnotetext{
${ }^{1}$ Paulus VI, Litterae apostolicae motu proprio datae „Apostolica sollicitudo” quibus Synodus Episcoporum pro universa Ecclesia constutuitur, 15 septembris 1965, in: AAS 57 (1965), p. 775-780. "Itaque, omnibus mature perpensis, pro Nostra existimatione atque observantia erga
} 
Dopo aver terminato il Concilio Vaticano II, il Sinodo dei Vescovi rimane un'istituzione che, avendo le radici nel Concilio, forma l'immagine della Chiesa post-conciliare. Perciò uno dei più significativi contributi del Concilio Vaticano II alla struttura organizzativa e al sistema amministrativo della Chiesa è indubbiamente proprio il Sinodo dei Vescovi, ciò viene confermato dal periodo post-conciliare insieme alle riunioni di questa istituzione che sono state già ventiquattro.

Bisogno anche aggiungere che oltre alle monografie in diverse lingue sul Sinodo dei $\mathrm{Vescovi}^{2}$, esiste anche la vasta bibliografia canonistica su questa centrale istituzione della Chiesa postconciliare ${ }^{3}$.

universos catholicos Episcopos, atque ut iisdem copia detur manifestiore efficacioreque ratione participandi sollicitudinem Nostram erga Ecclesiam universam, motu proprio ac Nostra apostolica auctoritate erigimus ac constituimus hac in alma Urbe stabile Episcoporum consilium pro Ecclesia universa, Nostrae potestati directe atque immediate subiectum, quod nomine proprio SYNODUM EPISCOPORUM appellamus". Ibid., p. 776.

${ }^{2}$ M. Alcala, Historia del Sinodo de los Obispos, Madrid 1996, BAC; J.I. Arrieta, El Sinodo de los Obispos, Pamplona 1987, EUNSA; M.C. Bravi, Il Sinodo dei Vescovi. Istituzione, fini e natura. Indagine teologico-giuridica, Roma 1995, Pontificia Università Gregoriana; A. Fernandez, Nuevas estructuras de la Iglesia, Burgos 1980, Aldecoa; F. Dupré La Tour, Le Synode des Évêques dans le contexte de la collégialité, Romae 2002, Parole et silence; D.R. Foley, The Synod of Bishops: its canonical structure and procedures, Washington D.C. 1973, UMI; A. Indelicato, Il Sinodo dei Vescovi. La collegialità sospesa 1965-1985, Bologna 2008, Il mulino; G.P. Milano, Il Sinodo dei Vescovi, Milano 1985, Giuffrè; T. Rozkrut, Instytucja Synodu Biskupów w Kościele posoborowym, Tarnów 2010, Biblos.

${ }^{3}$ A. Antón, Episcoporum Synodus: partens agens totius catholici Episcopatus, „Periodica” 57 (1968), p. 495-527; A. Antón, La collegialità nel Sinodo dei Vescovi, in: J. Tomko (a cura di), Il Sinodo dei Vescovi. Natura, metodo, prospettive, Città del Vaticano 1985, Libreria Editrice Vaticana, p. 59-111; A. Antón, Verso una collegialità più effetiva nel Sinodo dei vescovi (I), „La rivista del clero italiano” 64 (1983) n. 4, p. 290-302; J.I. Arrieta, Il Sinodo dei Vescovi. Rilevanza della prassi di attuazione in ordine al compimento delle sue finalità istituzionali, in: Pontificium Consilium de Legum Textibus Interpretandis, Ius in vita et in Missione Ecclesiae. Acta Symposii Internationalibus Iuris Canonici occurrente X anniversario promulgationis Codicis Iuris Canonici diebus 19-24 Aprilis 1993 in Civitate Vaticana Celebranti, Città del Vaticano 1994, Libreria Editrice Vaticana, p. 309323; J.I. Arrieta, Il sinodo dei vescovi quale istituto di comunione, „Ius Ecclesiae” 3 (1991) n. 2, p. 356-365; J.I. Arrieta, Lo sviluppo istituzionale del Sinodo dei Vescovi, „Ius Ecclesiae” 4 (1992) n. 1, p. 189-213; W. Bertrams, Commentarium in Litteras Apostolicas «Apostolica sollicitudo» Pauli Papae VI, „Periodica de re morali canonica liturgica” 55 (1966) n. 1, p. 115-132; W. Bertrams, De Synodi Episcoporum potestate cooperandi in exercitio potestatis primatialis „Periodica” 57 (1968) n. 3, p. 528-549; W. Bertrams, Struttura del «Sinodo dei Vescovi», „La civiltà Cattolica” 116 (1965) n. 4, p. 417-423; G. Caprile, Il Sinodo dei Vescovi e il suo funzionamento, in: J. Tomko (a cura di), Il Sinodo dei Vescovi. Natura, metodo, prospettive, Città del Vaticano 1985, Libreria Editrice Vaticana, p. 121-157; P. Colella, Collegialità episcopale e Sinodo dei Vescovi, in: AA. VV., Atti del Congresso Internazionale di Diritto Canonico. La Chiesa dopo il Concilio, t. 2, Milano 1972, Giuffrè, p. 335-350; C. Colombo, Il Sinodo episcopale, „La rivista del Clero Italiano” 49 (1968), p. 5-6; J. Dyduch, Kardynat Karol Wojtyła w stużbie Kościołowi Powszechnemu. Udział w pracach Kurii 
Il Sinodo dei Vescovi ha svolto e svolge ancora un importante ruolo anche

Rzymskiej i Synodów Biskupów, Kraków 1998, Wydawnictwo św. Stanisława BM Archidiecezji Krakowskiej; J. Dyduch, Wkład kardynała Karola Wojtyły w dzieło I Synodu Biskupów 1967 r., „Prawo Kanoniczne” 39 (1996) n. 3-4, p. 155-169; E. Erdö, Aspetti giuridici del Sinodo dei Vescovi. Quattro decenni di sviluppo istituzionale, in: N. Eterović (ed.), Il Sinode dei Vescovi. 40 anni di storia 1965-2005, Città del Vaticano 2005, Lateran University Press, p. 39-55; N. Eterović (a cura di), Il Sinodo dei Vescovi. 40 anni di storia 1965-2005, Città del Vaticano 2005, Lateran University Press; N. Eterović, Sinodo dei Vescovi: espressione privilegiata della comunione episcopale, in: N. Eterović (a cura di), Il Sinode dei Vescovi. 40 anni di storia 1965-2005, Città del Vaticano 2005, Lateran University Press, p. 23-26; M. Faccani, Collegio e collegialità episcopali nel Sinodo 1969, Bologna 1991, EDB; V. Fagiolo, Il Synodus Episcoporum, „Ephemerides Iuris Canonici” 25 (1969) n. 1-2, p. 9-64; V. Ferrara, Il Sinodo dei Vescovi tra ipotesi e realtà „Apollinaris” 42 (1969) n. 1-2, p. 491-555; M. Francescato, Strutture centrali della Chiesa universale, in: AA. VV., Il diritto nel mistero della Chiesa, t. 2, Roma 1990, Pontificia Università Lateranense, p. 555588; C.G.Fürst, Die Bischofssynode, in: J. Listl i H. Schmitz (ed.), Handbuch des katholischen Kirchenrechts, Regensburg 1999, F. Pustet, p. 353-359; S. Gądecki, Stowo Boże w życiu i misji Kościoła. Refleksje na kanwie XII Generalnego Zgromadzenia Zwyczajnego Synodu Biskupów w Rzymie (5-26 październik 2008 r.) „Currenda” 1 (2009), p. 133-146; G. Ghirlanda, Adnotatio ad responsum authenticum circa can. 346 \& 1 „Periodica” 81 (1992), p. 347-350; E. Górecki, Jan Pawet II a Synod Biskupów w latach 1978-2003, in: S. Tymosz (a cura di), Synodalność Kościoła w nauczaniu i działalności Jana Pawła II. Materiały ogólnopolskiej sesji naukowej zorganizowanej 23 października 2003 r. w Lublinie, Lublin 2004, Wydawnictwo KUL, p. 15-34; E. Górecki, Synod Biskupów, in: J. Krukowski (ed.), Komentarz do Kodeksu Prawa Kanonicznego, Poznań 2005, Pallottinum, v. 2/1, p. 174-184; J.L. Gutiérrez, Il Sinodo dei Vescovi, in: J.I. Arrieta (ed.), Codice di Diritto Canonico e leggi complementari commentato, Roma 2004, Coletti a San Pietro Editore, p. 283-288; J. Hamer, I soggetti della suprema potestà nella Chiesa: visione teologica „Apollinaris” 61 (1983), p. 475-485; J.G. Johnson, The Synod of Bishops: an Exploration of its Nature and Function, „Studia Canonica” 20 (1986), p. 275-318; F.B. Kloppenburg, O Sinodo dos Bispos. Representação e Ação do Colégio?, „Revista Eclesiástica Brasileira” 27 (1967) n. 2, p. 339-347; A. Marranzini, Il Sinodo organo della «sollecitudine pastorale dei vescovi» per il popolo di Dio, „La rivista del clero italiano” 64 (1983) n. 11, p. 847-858; A. Marranzini, Sinodo dei Vescovi e collegialità, in: J. Tomko (a cura di), Il Sinodo dei Vescovi. Natura, metodo, prospettive, Città del Vaticano 1985, Libreria Editrice Vaticana, p. 112-120; G.P. Milano, De Synodo Episcoporum, in: A. Marzoa, J. Miras, R. Rodríguez-Ocańa (a cura di), Comentario exegético al Código de derecho canónico, Pamplona 1996, EUNSA, v. 2, p. 603-625; G.P. Milano, Il Sinodo dei Vescovi: natura, funzioni, rappresentatività „L'année canonique, hors série” (1992), v. 1, p. 167-182; G.P. Milano, Paolo VI e il principio di collegialità nel Sinodo dei Vescovi, in: AA. VV., Paul VI et les réformes institutionnelles dans l'Église, Brescia 1987, Pubblicazioni dell'Istituto Paolo VI, p. 30-48; S. Nagy, Doktryna Synodu, ,Analecta Cracoviensia” 2 (1970), p. 157-187; J.H. Provost, The Synod of Bishops, in: J.A. Coriden, T.J. Green, D.E. Heintschel (a cura di), The Code of Canon Law. A text and Commentary, New York 1985, Paulist Press, p. 281-286; J.P. Schotte, The Synod of Bishops: A Permanent yet Adaptable Church Institution „Studia Canonica” 26 (1992), p. 289-306; R. Sobański, Il concilio ecumenico. Il sinodo dei vescovi. Il collegio cardinalizio, in: A. Longhitano (a cura di), Collegialità e primato. La suprema autorità della Chiesa, Bologna 1993, EDB, p. 69-124; S. Sołtyszewski, Synod Biskupów, „Prawo Kanoniczne” 9 (1966) n. 3-4, p. 287-300; O. Stoffel, Die Bischofssynode, in: K. Lüdicke (a cura di), Münsterischer Kommentar zum Codex Iuris Canonici, v. 2 (cann. 204-459), Essen 1998, Ludgerus; E. Sztafrowski, Synod Biskupów nowym organem kolegialnym, „Prawo Kanoniczne” 16 (1973) n. 3-4, p. 111-169; A. Talamanca, Sinodo dei Vescovi, in: Enciclopedia giuridica, v. 27, Roma 1992, Il veltro, p. 1-7; J. Tomko, Aspetti teologici 
durante i pontificati dei papi post-conciliari, in particolare negli anni del lungo pontificato di Giovanni Paolo II (1978-2005), anche il pontificato attuale dimostra che il papa Benedetto XVI dà molta importanza a questa istituzione convocando regolarmente il Sinodo dei Vescovi. Del resto anche prima il cardinale J. Ratzinger ha partecipato a molti Sinodi dei Vescovi, molto significativi sono anche i suoi interventi dottrinali sull'istituzione del Sinodo dei Vescovi ${ }^{4}$. Similmente il cardinale K. Wojtyła prima di essere stato nominato il Vescovo di Roma il 16 ottobre 1978, aveva partecipato ai dibattimenti del Sinodo dei Vescovi, cercando nello stesso tempo di informarne l'opinione pubblica in Polonia, cosi trasmetteva anche le informazioni sulla natura e missione del Sinodo dei Vescovi, ciò senza dubbio influiva la formazione della Chiesa post-conciliare anche in Polonia ${ }^{5}$.

Risultato delle esperienze sinodali sono state, tra l'altro dieci Esortazioni Apostoliche post-Sinodali delle Assemblee Generali (Evangelii nuntiandi 1975, Catechesi tradendae - 1979, Familiaris consortio - 1981, Reconciliatio et paenitentia - 1985, Christifideles laici - 1988, Pastores dabo vobis - 1992, Vita consecrata - 1996, Pastores gregis - 2003, Sacramentum caritatis - 2007, Verbum Domini -2010), sei Esortazioni Apostolice post-Sinodali delle Assemblee Speciali (Ecclesia in Africa - 1995, Une espérance nouvelle pour le Liban-1997, Ecclesia in America - 1999, Ecclesia in Asia - 1999, Ecclesia in Oceania -2001, Ecclesia in Europa - 2003).

Inoltre bisogna ricordare numerose proposte operative del Sinodo dei Vescovi, accolte dal Romano Pontefice, che hanno avuto seguito nell'istituzione della Commissione Teologica Internazionale (Sinodo dei Vescovi del 1967), nel consenso raggiunto sulle norme da osservare nella promulgazione di Codice di Diritto Canonico del 1983 (Sinodo del 1967), nella richiesta di preparare un

del Sinodo dei Vescovi. Dottrina e dinamismo, in: N. Eterović (a cura di), Il Sinode dei Vescovi. 40 anni di storia 1965-2005, Città del Vaticano 2005, Lateran University Press, p. 27-37; J. Tomko, Il Sinodo dei Vescovi e Giovanni Paolo II, in: J. Tomko (a cura di), Il Sinodo dei Vescovi. Natura, metodo, prospettive, Libreria Editrice Vaticana 1985, Città del Vaticano, p. 13-44; J. Tomko (a cura di), Il Sinodo dei Vescovi. Natura, metodo, prospettive, Libreria Editrice Vaticana 1985, Città del Vaticano; A.G. Urru, Istituti per l'esercizio della collegialità e del primato: il Concilio Ecumenico e il Sinodo dei Vescovi, „Monitor Ecclesiasticus” 115 (1990) n. 4, p. 569-589; A. Viana, Las nuevas normas estatutarias del Sínodo de los Obispos, „Ius Canonicum” 94 (2007), p. 657-676; G. Zizola, Il Sinodo dei vescovi. Cronaca - bilancio - documentazione, Torino 1968, Borla; M. Żurowski, Synodus Episcoporum in quantum «partens agens totius catholici episcopatus», „Periodica de re morali canonica liturgica" 62 (1973) n. 3, p. 375-391.

${ }^{4}$ J. Ratzinger, Scopi e metodi del Sinodo dei Vescovi, in: J. Tomko (a cura), Sinodo dei Vescovi. Natura, Metodo, Prospettive, Città del Vaticano 1985, Libreria Editrice Vaticana, p. 45-58.

${ }^{5}$ K. Wojtyła, O Synodzie Biskupów, in: „Tygodnik Powszechny”, 5 marzec 1972, p. 1 e 5; K. Wojtyła, Synod Biskupów: Zebranie Nadzwyczajne Rzym 1969, „Analecta Cracoviensia” 2 (1970), p. 131-156. 
Catechismo della Chiesa universale (Sinodo dei Vescovi del 1985), una Carta dei Diritti della famiglia (Sinodo dei Vescovi del 1980), un Compendio della Dottrina Sociale della Chiesa (Sinodo dei Vescovi del 1985), un aggiornamento del Direttorio per il Ministero Pastorale dei Vescovi (Sinodo dei Vescovi del $2001)^{6}$.

I Sinodi hanno fornito indicazioni circa l'aplicazione concreta della riforma liturgica, il rinnovamento della formazione nei Seminari, la cooperazione tra la Santa Sede e le Conferenze Episcopali, come pure su vari problemi pastorali nelle mutevoli condizioni della comunità umana ${ }^{7}$.

I papi della Chiesa post-conciliare menzionati sopra (Paolo VI, Giovanni Paolo II, Benedetto XVI) hanno formato anche un'immagine teologica e giuridica del Sinodo dei Vescovi. Prima di tutto in questa corrente si devono ricordare: m.p. „Apostolica sollicitudo" di Paolo VI che ha costituito un'istituzione la quale fino ad oggi è un perno teologico-giuridico del Sinodo dei Vescovi, e in seguito la formazione dell'attuale aspetto giuridico dal Codice di Diritto Canonico promulgato dal papa Giovanni Paolo II nel 1983 insieme alla divisione nelle Riunioni Generali (ordinarie e straordinarie) e la Riunione Speciale ${ }^{8}$.

In seguito occorrerebbe enumerare i regolamenti di questa istituzione centrale della Chiesa e cioè il Regolamento del Sinodo dei Vescovi degli anni: 1966 $1969^{10}, 1971^{11}$ e $2006^{12}$.

Il Regolamento del Sinodo dei Vescovi ha formato in maniera pratica i lavori delle sue singole congregazioni. In questo ambito si devono anche nominare i discorsi dei Vescovi di Roma riguardanti diversi problemi in diverse circostanze sul Sinodo dei Vescovi ${ }^{13}$.

${ }^{6}$ T. Rozkrut, op. cit., p. 212-234.

${ }^{7}$ N. Eterović, op. cit., p. 25.

8 „Il sinodo dei Vescovi può riunirsi in assemblea generale ordinaria o straordinaria, in cui vengono trattati argomenti che riguardano direttamente il bene della Chiesa universale, oppure può riunirsi in assemblea speciale, in cui vengono trattati affari che riguardano direttamente una o più regioni determinate", CIC'83, can. 345.

${ }^{9}$ Ordo Synodi Episcoporum celebrandae, AAS 59 (1967), p. 91-103.

${ }^{10}$ Ordo Synodi Episcoporum celebrandae recognitus et auctus, AAS 61 (1969), p. 525-539.

${ }^{11}$ Ordo Synodi Episcoporum celebrandae recognitus et auctus nonnullis additamentis perficitur, AAS 63 (1971), p. 702-704.

${ }^{12}$ Ordo Synodi Episcoporum, AAS, 98 (2006) p. 755-779.

${ }^{13}$ Benedetto XVI, Meditazione del Santo Padre durante l'Ora Terza del 3 ottobre 2005, in: N. Eterović (a cura di), Il Sinode dei Vescovi. 40 anni di storia 1965-2005, Città del Vaticano 2005, Lateran University Press, p. 11-16; Giovanni Paolo II, Discorso al Consiglio della Segreteria Generale del Sinodo dei Vescovi (30 Aprile 1983), in: J. Tomko (a cura di), Il Sinodo dei Vescovi. Natura, metodo, prospettive, Città del Vaticano 1985, Libreria Editrice Vaticana, p. 9-12; Giovanni 


\section{Il breve testo ha intenzione di indicare come il Sinodo dei Vescovi ha formato}

Paolo II, Lettera "Dal santuario di Fatima" ai vescovi europei circa la preparazione del sinodo per l'Europa, in: Segreteria Generale del Sinodo dei Vescovi (a cura di), Enchiridion del Sinodo dei Vescovi, v. 2, Bologna 2006, EDB, p. 3324-3326; Ioannes Paulus II, Ad Episcopos, Sacerdotes et Christifideles totius Ecclesiae Catholicae: suplicationes pro VI Synodo Episcoporum indicuntur, AAS 72 (1980), p. 791-793; Ioannes Paulus II, Ad Patres Cardinales, Familiam domni Papae Romanamque Curiam, imminente Nativitate Domini Iesu Christi habita, AAS 83 (1991), p. 740749; Ioannes Paulus II, Allocutio "Deo Gratias" ago in conclusione secundae coetus generalis extraordinariae Synodi episcoporum, AAS 78 (1986), p. 431-438; Ioannes Paulus II, Allocutio "Gesù in persona" ineunte Synodo episcoporum Europae, AAS 92 (2000), p. 173-176; Ioannes Paulus II, Allocutio "II Gesù" il Nazareno Synodo episcoporum pro Oceania ineunte, AAS 91 (1999), p. 441-444; Ioannes Paulus II, Allocutio "Gratias agamus" VII exeunte ordinario generali coetu Synodi Episcoporum habita, AAS 80 (1988), p. 606-611; Ioannes Paulus II, Allocutio "Laetus, vobiscum communicans" in XXX et postrema congregatione generali VIII Coetus ordinarii Synodi episcoporum habita, AAS 83 (1991), p. 494-499; Ioannes Paulus II, Allocutio "Misericordias Domini" exeunte VI Synodi episcoporum generali coetu habita, AAS 76 (1984), p. 281-289; Ioannes Paulus II, Allocutio "Questo è il giorno" ineunte Synodo episcoporum pro Africa habita, AAS 87 (1995), p. 179-185; Ioannes Paulus II, Allocutio "Signore ... tu hai parole" ineunte speciali coetu pro Europa Synodi Episcoporum habita, AAS 84 (1992), p. 1148-1151; Ioannes Paulus II, Allocutio "Vegliate e state pronti" Synodo episcoporum pro America ineunte, AAS 90 (1998), p. 680-688; Ioannes Paulus II, Homilia "Expedit, ut laborum" in Xystino Sacello habita, VI Synodo episcoporum ineunte, AAS 72 (1980), p. 1007-1011; Ioannes Paulus II, Synodo extraordinaria exeunte ad Patres congregatos habita, AAS 78 (1986), p. 431-438; Ioannes Paulus II, Universis Europae episcopis missus, AAS 84 (1992), p. 163-168; John Paul II, Omily "Quello che vedi" in Mass for the opening of Special Assembly for Asia, in: Segreteria Generale del Sinodo dei Vescovi (a cura di), Enchiridion del Sinodo dei Vescovi, t. 3, Bologna 2008, EDB, p. 57905798; Paulus VI, Ad E.mos Patres Cardinales, Exc.mos Praesules, Rev.mos Praelatos ceterosque Romanae Curiae Officiales, AAS 55 (1963), p. 793-800; Paulus VI, Ad E.mos Patres Cardinales, ad Romanae Curiae Pontificalisque Domus Praelatos, per E.mum Sacri Collegii Decanum Summo Pontifici felicia ac fausta ominatos, Nativitatis Domini nostri Iesu Christi festo recurrente, AAS 61 (1969), p. 34-46; Paulus VI, Allocutio „Deo Patri omnipotenti” patribus et praesulibus e Synodo episcoporum, prima coacto coetui operam navantibus, AAS 59 (1967), p. 969-975; Paulus VI, Allocutio „Ecce ad nostrae” patribus cardinalibus et praesulibus e Synodo episcoporum, cum tertius generalis coetus exitum haberet, AAS 66 (1974), p. 631-639; Paulus VI, Allocutio „, Gratia vobis et pax" in patriarchali Basilica Vaticana habita, eucharistico sacrificio concelebrato, ut episcoporum Synodi coetus inaugurarentur, AAS 59 (1967), p. 963-969; Paulus VI, Allocutio habita in Vaticana Basilica ad Patres Conciliares, in festo Exaltationis Crucis Domini nostri Iesu Christi, cum quarta Concilii Oecumenici Vaticani II Sessio initium caperet, solemni ab ipso Summo Pontifice peracta Missae concelebratione, AAS 57 (1965), p. 804; Paulus VI, Allocutio in auditorio Urbanianae Studiorum Universitatis die rei missionali provehendae per totum Orbem dicato recurrente, AAS 66 (1974), p. 625-629; Paulus VI, Allocutio „Laeto animo” ineunte quarto coetu generali ordinario Synodi Episcoporum, vespere in synodi aula habita, AAS 69 (1977), p. 629631; Paulus VI, Allocutio „Synodum hanc extraordinariam” patribus cardinalibus et praesulibus e Synodi episcoporum, laboribus iam ad finem vergentibus, AAS 61 (1969), p. 726-730; Paulus VI, Constitutio apostolica „Regimini ecclesiae universae”, AAS 59 (1967), p. 885-928; Paulus VI, E.mis Patribus Cardinalibus et Exc.mis Praesulibus e Consilio Secretariae generalis Synodi Episcoporum, qui Romae primo Coetui interfuerunt, AAS 62 (1970), p. 443-446; Paulus VI, In Sede Sixtina habita a Beatissimo Patre Sacrum concelebrate, ineunte Extraordinaria Episcoporum Synodo, AAS 61 (1969), p. 716-721. 
234 Volume 1(2011) Number 2

la vita della Chiesa post-conciliare. Naturalmente c'è presente anche un elemento teologico del Sinodo dei Vescovi, però deve essere percepito come ausiliare conformemente all'insegnamento del Concilio Vaticano II, secondo il quale l'interpretazione del diritto canonico dovrebbe riferirsi al mistero della Chiesa ai sensi della Costituzione dogmatica sulla Chiesa Lumen gentium. Questo elemento, cioè l'ecclesiologia conciliare, è necessario per conoscere le origini del Sinodo dei Vescovi e per capire la natura e il ruolo dell'istituzione nella Chiesa post-conciliare ${ }^{14}$.

\footnotetext{
${ }^{14}$ T. Rozkrut, op. cit., p. 99-153.
} 